\title{
Análise das práticas de produtores em sistemas de produção leiteiros e seus resultados na produção e qualidade do leite
}

\author{
Farmer's conduct analisys in dairy systems and its results in \\ production and quality of milk
}

\author{
José Francisco Lopes Junior ${ }^{1}$; Carlos Eduardo Crispim de Oliveira Ramos²; \\ Geraldo Tadeu dos Santos ${ }^{3 *}$; Paula Adriana Grande ${ }^{4}$; \\ Julio Cesar Damasceno ${ }^{3}$; Ely Mitie Massuda ${ }^{5}$
}

\section{Resumo}

Com o objetivo de caracterizar 18 sistemas de produção leiteiros quanto à gestão zootécnica e resultados para produção e qualidade do leite, foi conduzido um estudo na região noroeste do estado do Paraná, em cinco municípios. Os dados de gestão dos sistemas foram colhidos via entrevista, orientada pelo método do diagnóstico rápido participativo e os dados da qualidade e produção do leite foram obtidos a partir de amostragens desses sistemas e posteriormente analisados em laboratório. Foram utilizadas para a análise dos dados técnicas de estatística multivariada; Análise de Correspondências múltiplas ACM e a Análise Classificatória Hierárquica. Obtiveram-se quatro grupos homogêneos de produtores quanto às estratégias de gestão zootécnica influenciando na produção de leite, de acordo com a análise de variância, tendo os grupos como fatores e os indicadores de qualidade e produção como variáveis resposta. Foram detectados diferentes perfis de ação quanto ao manejo da ordenha, estrutura da propriedade, porém as diferenças mais marcantes entre grupos foram quanto ao nível tecnológico e continuidade da atividade em função do perfil etário dos grupos. As ações sobre os grupos (ATER), portanto, devem ser direcionadas ao perfil de cada grupo e não homogeneizadas como é usual, para atingir a todos os sistemas e viabilizá-los.

Palavras-chave: Diversidade, variáveis qualitativas, permanência no campo

\begin{abstract}
There was carried a research with 18 dairy systems aiming typify these farms concerning their zootechnical conduct and comparing that against the production and quality indicators of the milk as a product. The study was placed in northwest Paraná state region, in five municipalities. Data was obtained using DRP - Fast Participative Diagnosis, by means of an interview. Milk was sampled in each farm and analyzed as follows. Data analysis was made using a multivariate techniques Multilple Correspondence Analysis and Cluster's Analysis. Were obtained four homogeneous groups (clusters) concerning to conduct that influenced $(\mathrm{p}<0,05)$ the quality and production indicators of the milk. This was obtained using analysis of variance with clusters as factors and milk variables as response variables. There were identified four distinct dairy farmer profiles. These concerning to: general structure of the
\end{abstract}

${ }^{1}$ Extensionista da Emater, Nova Esperança, PR. E-mail: chicojunior_lopes@yahoo.com.br

2 Pós-Doutorando no Programa de Pós-Graduação em Zootecnia da Universidade Estadual de Maringá, UEM, Bolsista DTI do Projeto Repensa/CNPq, Maringá, PR. E-mail: jaguaricarlos@gmail.com

3 Profs. do Dept ${ }^{0}$ de Zootecnia da UEM, Maringá, PR. E-mail: gtsantos@uem.br; jcdamasceno@uem.br

${ }^{4}$ Pós-Doutoranda no Programa de Pós-Graduação em Zootecnia da UEM, Bolsista DTI - Ed40/2008 CTAgro/CNPq/MAPA. Cadeias Produtivas do Leite e da Carne. Maringá, PR. E-mail: paulagrde@gmail.com

5 Prof a do Centro de Ensino Superior de Maringá, CESUMAR, Maringá, PR. E-mail: elymitie@hotmail.com

* Autor para correspondência 
dairy system, technologic level and possibility of continuity of dairy farming related to farmer's age. The actions of technical assistance must be adequate to those profiles but not homogenized, like is usual in our context, for reach many dairy farms as possible and make these production systems.

Key words: Strategy diversity, synthetic variables, milk production, rural exodus

\section{Introdução}

Entre as características da pecuária leiteira nacional, duas merecem destaque. A primeira é que a produção ocorre em todo o território nacional, em mais de um milhão de propriedades rurais e, somente na produção primária, gera acima de três milhões de empregos e agrega mais de $\mathrm{R} \$ 6$ bilhões ao valor da produção agropecuária nacional (VILELA; LEITE; RESENDE, 2002). Existe informação de produção de leite em 554 microrregiões, das 558 consideradas pelo IBGE - Instituto Brasileiro de Geografia e Estatística. A segunda característica é que não existe padrão na produção. Existem desde propriedades de subsistência, com produção diária menor do que dez litros, até produtores com tecnificação e produção diária superior a 60 mil litros (ZOCCAL; GOMES, 2005).

Este cenário de diversidade e de heterogeneidade dos sistemas de produção leiteiros SPL, contribui para o desconhecimento da real situação da atividade por parte dos gestores públicos e para a inadequação, ou então a padronização do conselho técnico por meio das agências de ATER - Assistência Técnica e Extensão Rural.

Segundo Hostiou et al. (2006) os SPL são, cada vez mais, reconhecidos como um objeto de estudo complexo necessitando, por este motivo de métodos de abordagem que levem em conta essa característica. $\mathrm{O}$ tratamento adequado às informações provenientes da realidade dos SPL visa gerar conhecimento científico aplicável (DAMASCENO et al., 2008).

Um importante aspecto dos sistemas de produção leiteiros a ser estudado e mapeado é o controle da qualidade. Este se inicia na base do processo de produção, que são as propriedades leiteiras, influenciando o desempenho de toda a cadeia.
Os padrões mínimos de qualidade são definidos e regulamentados pela Instrução Normativa $n^{0}$ 51 do Ministério da Agricultura e Abastecimento (BRASIL, 2002). Há autores ainda que detalhem os aspectos de qualidade do leite, bem como os procedimentos de determinação desta (TRONCO, 2008). O entendimento central na qualidade do leite é o fato de que ela é construída por uma série de práticas, desde a ordenha até o envasamento e consumo. Partindo deste princípio podem-se aplicar métodos de análise que apontem os pontos críticos da qualidade do leite baseado na natureza dos sistemas e ainda mapeiem esses sistemas quanto à intensidade e efetividade das ações para obter-se um produto de qualidade.

Análise de correspondência múltipla (ACM) é uma das técnicas da análise estatística multivariada, que apresenta capacidade de análise para obter esse tipo de informação de variáveis que descrevem a gestão em um sistema de produção leiteira. Desta forma o objetivo principal neste trabalho, é o de propor bases teóricas para auxiliar o aconselhamento e as decisões técnicas sobre a atividade leiteira, levando em conta aspectos de qualidade do leite.

\section{Material e Métodos}

Esse estudo foi realizado em 18 (dezoito) SPL, situados nos municípios de Atalaia, Floraí, Nova Esperança, Presidente Castelo Branco e Alto Paraná; região Noroeste do Estado do Paraná. O período de estudo foi de outubro de 2008 a outubro de 2009. As propriedades escolhidas fazem parte do Projeto Vitória, desenvolvido e coordenado pelo Instituto Emater-PR.

As informações para a caracterização dos SPL foram obtidas junto aos produtores, utilizando o DRP - Diagnóstico Rápido Participativo 
(VERDEJO, 2006; VALLADARES, 2007). Foram aplicados questionários-guia semi-estruturados. As questões foram elaboradas com a finalidade de colher informações gerais do produtor, dados sobre o rebanho (nutrição e sanidade), produção de leite e ordenha, estrutura produtiva, meio ambiente e gestão. Esses dados serviram de base para a posterior construção das variáveis explicativas (LEBART; MORINEAU; TABARD, 2000).

As informações acerca da qualidade de leite foram obtidas via colheita de amostras de leite em cada propriedade, logo após a ordenha, uma vez ao mês. Em cada coleta uma amostra de cada vaca, foi colhida e homogeneizada formando uma única amostra por vaca. Essas amostras foram acondicionadas em frascos plásticos e preservadas com 2-bromo-2-nitropano-1,3diol (Bronopol). As amostras de leite foram analisadas no Laboratório do Programa de Análises do Rebanho Leiteiro do Paraná (PARLPR) pertencente à Associação Paranaense de Criadores de bovinos da Raça Holandês (APCBRH), em Curitiba.

A avaliação da composição química do leite foi feita pela determinação da proteína, gordura, lactose e sólidos totais determinados por meio de espectrofotometria por radiação infravermelha no equipamento Bentley $2000^{\circledR}$, conforme descrito em Souza et al. (2004), assim como a contagem de células somáticas. As análises do nitrogênio ureico no leite foram feitas por um analisador infravermelho.

Todas as vacas das propriedades foram submetidas aos exames de Brucelose e Tuberculose. Somente permaneceram nas propriedades avaliadas as vacas que apresentaram exames negativos para ambas as doenças.

A determinação de variáveis explicativas da diversidade do sistema de produção foi feita por meio de uma técnica de estatística multivariada, para dados qualitativos denominada: Análise de Correspondência Múltipla - ACM (LEBART; MORINEAU; TABARD, 2000; SMITH; MOREIRA; LATRILLE, 2002). Para determinar as variáveis de eleição foi utilizada a variância explicada (inércia) para o modelo completo da ACM como critério. As variáveis originais foram comparadas por meio da correlação e em seguida foi feita uma análise hierárquica ascendente (ou cluster) com a finalidade de agrupar os SPL de acordo com as práticas características realizadas para a qualidade do leite. Por último os clusters (ou grupos de SPL) foram considerados como fatores e analisados contra as variáveis de qualidade do leite (produção de leite, gordura, proteína, lactose, sólidos totais, contagem de células somáticas e nitrogênio ureico do leite), consideradas variáveis dependentes, por meio de uma ANOVA - Análise de Variância. As médias das diferenças entre grupos obtidas foram submetidas ao teste Tukey com significâncias $\mathrm{p}<0,05$ e $\mathrm{p}<0,01$. Foi utilizado o software Statistical Package for the Social Sciences (SPSSS 18.0) para análise dos dados.

\section{Resultados e Discussão}

Os SPL estudados apresentaram uma diversidade de comportamentos quanto às variáveis definidas, isso é denotado pela sua relação com as variáveis sintéticas (Dim1 e Dim2) mostradas na Tabela 1.

A contribuição de cada caso é dada pela incidência de cada variável dentro do caso, ou seja, como determinada ação produtiva é realizada naquele SPL. O peso que isso tem para determinar o perfil das unidades produtoras de leite é medido pela inércia. Este perfil do SPL "propriedade" é característico, e é determinado pelo grau de correlação que existe entre cada sistema e as dimensões (Dim1; Dim2). Para exemplificar pode-se citar a propriedade 18 (P18) está mais relacionada à dimensão $1(\mathrm{Dim} 1=$ $0,255)$ do que com a $2(\operatorname{Dim} 2=0,027)$. 
Tabela 1. Estatísticas da análise de correspondência múltipla (ACM), e contribuições de explicação (inércia) para os 18 sistemas de produção estudados para as duas dimensões do plano fatorial (Dim1 e Dim2)

\begin{tabular}{ccc}
\hline Casos (SPL) & \multicolumn{2}{c}{ Inércia caso** } \\
\cline { 2 - 3 } & DIM1 & DIM2 \\
P1 & 0,078 & 0,121 \\
P2 & 0,032 & 0,112 \\
P3 & 0,018 & 0,000 \\
P4 & 0,040 & 0,212 \\
P5 & 0,056 & 0,003 \\
P6 & 0,192 & 0,007 \\
P7 & 0,156 & 0,205 \\
P8 & 0,225 & 0,027 \\
P9 & 0,005 & 0,079 \\
P10 & 0,042 & 0,002 \\
P11 & 0,000 & 0,026 \\
P12 & 0,003 & 0,077 \\
P13 & 0,045 & 0,026 \\
P14 & 0,010 & 0,020 \\
P15 & 0,002 & 0,020 \\
P16 & 0,034 & 0,002 \\
P17 & 0,012 & 0,022 \\
P18 & 0,050 & 0,039 \\
\hline
\end{tabular}

"Casos" aqui se referem às propriedades leiteiras pesquisadas (P1, P2, P3..., P18); Inércia; **Contribuição de cada caso à respectiva dimensão em termos de inércia; DIM1 = dimensão 1; DIM2 = dimensão 2.

Fonte: Dados dos autores.

Quando se fala em dimensões é fundamental entender a noção de variável sintética, (BARROSO; ARTES, 2003; LEBART; MORINEAU; TABARD, 2000) representada por estas, para que se possam visualizar as informações dadas pela ACM sobre a natureza dos sistemas de produção.

As dimensões são um meio de se resumir todas as variáveis estudadas, originalmente colhidas nas propriedades, por isso a denominação "sintética". $\mathrm{Na}$ Tabela 2 observam-se as quatro primeiras dimensões que resumem a análise dos dados, sendo que foram utilizadas as primeiras três para os presentes resultados, segundo o critério da variância explicada em torno de 60\% (BARROSO; ARTES, 2003).

Tabela 2. Estatísticas da ACM e contribuições de inércia para as dimensões formadas das variáveis originais

\begin{tabular}{lcccc}
\hline \multicolumn{5}{c}{ Estatísticas das dimensões (ou fatores) da ACM } \\
\hline $\mathrm{N}$ & Autovalores & Inércia & \% Variância & Variância acumulada\% \\
\hline DIM1 & 7,746 & 0,484 & 48,41 & 48,41 \\
DIM2 & 3,643 & 0,228 & 22,77 & 71,18 \\
DIM3 & 3,081 & 0,193 & 19,26 & 90,44 \\
Total & 14,47 & 0,904 & - & - \\
\hline
\end{tabular}

$\mathrm{N}=$ Dimensões (DIM1a DIM4), as variáveis sintéticas.

Fonte: Elaboração dos autores. 
A percentagem acumulada da variância explicada pelos dois primeiros eixos é de $71,18 \%$, satisfazendo o critério acima. Observa-se que esse valor é alto comparado a Smith, Moreira e Latrille (2002) que com enfoque similar relataram, respectivamente, $24,00 \%$ de variância explicada em duas dimensões e 75,56\% em quatro. Este comportamento pode ser explicado pelo critério de escolha das variáveis de eleição que é responsável por balancear variância explicada e quantidade de informações incorporadas a cada variável sintética (LEBART; MORINEAU;
TABARD, 2000). O resultado final não é melhor e nem pior, dependendo do enfoque analítico: explicitar melhor os detalhes ou fazer um estudo mais global. Na Tabela 3 observa-se a grandeza das correlações entre as dimensões da ACM e as variáveis originais componentes de dimensões (da $1^{\mathrm{a}}$ a $3^{\mathrm{a}}$ ). Essa medida dá uma idéia da associação entre as variáveis qualitativas originais que medem ações dos produtores sobre os SPL e as dimensões formadas da síntese dessas ações, ou seja, as variáveis explicativas.

Tabela 3. Correlação entre os três primeiros fatores ou dimensões e as variáveis da ACM

\begin{tabular}{lcccc}
\hline Variáveis & Sigla & \multicolumn{3}{c}{ Dimensão } \\
\cline { 3 - 5 } & & 1 & 2 & 3 \\
\hline Área total & ATT & 0,294 & 0,398 & 0,43 \\
Idade do produtor & IPD & 0,512 & 0,515 & 0,037 \\
Vontade de melhorar & VTM & 0,397 & 0,188 & 0,097 \\
Mão de obra & MDO & 0,003 & 0,051 & 0,077 \\
Análise de solo & ANS & 0,376 & 0,629 & 0,756 \\
Capineira & CPE & 0,39 & 0,042 & 0,003 \\
Utiliza uréia & UNT & 0,671 & 0,183 & 0,028 \\
Raça (genética) & GEN & 0,45 & 0,09 & 0,483 \\
Pré-parto & PPT & 0,747 & 0,088 & 0,014 \\
Leite/litros/dia ${ }^{1}$ & LLD & 0,754 & 0,775 & 0,608 \\
Número de ordenhas & NOR & 0,188 & 0,032 & 0,158 \\
Veste do ordenhador & VST & 0,671 & 0,183 & 0,028 \\
Desinfecção pós-ordenha (Pós-dipping) & POD & 0,821 & 0,01 & 0,085 \\
Tipo ordenha & TOR & 0,564 & 0,013 & 0,056 \\
Piso do local da ordenha & POR & 0,454 & 0,224 & 0,112 \\
Ambiente confortável na ordenha & AMB & 0,454 & 0,224 & 0,112 \\
Ativo Total & & 7,746 & 3,643 & 3,081 \\
\% da Variância & & 48,41 & 22,766 & 19,259 \\
\hline
\end{tabular}

Fonte: Elaboração dos autores.

As variáveis que têm mais impacto na formação da dimensão 1 foram as que estão relacionadas ao nível tecnológico, ou seja, uma escala de adoção e investimento em tecnologia e qualidade do leite (utilização de adubos nitrogenados, realização de pré-parto, vestimenta apropriada para ordenha, prevenção de mastite e tipo de ordenha). Este é o primeiro aspecto importante para tipificar os casos em estudo, já que essas variáveis juntas respondem por $48,41 \%$ da variância.

$\mathrm{Na}$ segunda dimensão estão as variáveis que dizem respeito à tecnologia básica, escala de produção e área e indicadores de continuidade da atividade em longo prazo (Área total da 
propriedade, idade do produtor, preocupação com a fertilidade do solo e quantidade de leite produzida). Uma interpretação mais detalhada pode ser feita utilizando as informações contidas na Figura 1.

Uma abordagem prática das variáveis no plano fatorial permite extrapolar os 71,18\% da variância explicada (Tabela 2) em termos de ações realizadas que caracterizam os eixos: dimensões 1 e 2 .

A porção negativa da dimensão 1 é caracterizada por SPL que têm maior nível tecnológico no que diz respeito à produção de leite caracterizado por: LLD:5 (produção superior a 300 L.dia ${ }^{-1}$ ); UNT:1 (utilização mais intensa de adubos nitrogenados); PPT:1 (realização de manejo pré-parto); GEN:1 (genética especializada leiteira); PRD:1 e POD:1 (realização de desinfecção dos tetos pré e pósordenha). Além disso, há uma forte correlação - demonstrada graficamente pela proximidade geométrica em relação à dimensão 1 - da variável IDP:1 (produtores mais novos, 30 a 40 anos) com as citadas anteriormente o que leva a crer que produtores mais novos adotam mais tecnologia, embora a variável escolaridade nessa amostra não tenha apresentado importância significativa na ACM, ao contrário do observado por Solano et al. (2001). Para a porção positiva da dimensão 1 a incidência de níveis de ocorrência, bem como a inércia acrescentada por estas foi menos importante, sendo relacionada desta forma a menores níveis de tecnologia, segundo a escala de interpretação da dimensão como um todo.

Figura 1. Representação fatorial das dimensões da $\mathrm{ACM}$ e dos níveis de incidência das variáveis quanto às ações nos SPL. Área total (ATT), Idade do produtor (IPD), Vontade de melhorar (VTM), Mão de obra (MDO), Análise de solo (ANS), Capineira (CPE), Utiliza uréia (UNT), Raça /genética (GEN), Pré-parto (PPT), Leite/litros/dia (LLD), Número de ordenhas (NOR), Veste do ordenhador (VST), Desinfecção pós-ordenha / Pós-dipping (POD), Tipo ordenha (TOR), Piso do local da ordenha (POR), Ambiente confortável na ordenha (AMB)

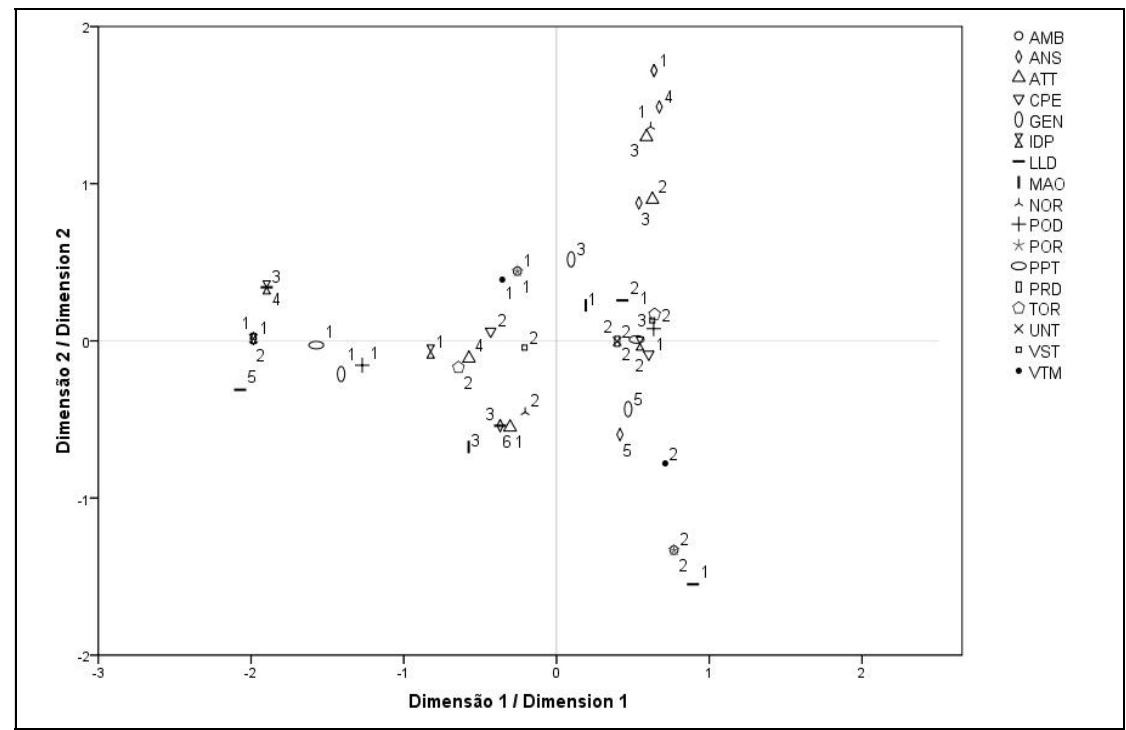

Fonte: Elaboração dos autores.

$\mathrm{Na}$ dimensão 2 a porção positiva foi marcada por indicadores de área de superfície (ATT:3, propriedades maiores de 24 ha) o que para estratificação da amostra representa área média a grande. Outro indicador foi NOR:1 (uma ordenha. $\mathrm{dia}^{-1}$ ) e ANS:4 (análise de solo em intervalos médios, em torno de 2 anos), o que caracteriza uma forma mais "extrativista" de exploração da superfície e 
também uma estratégia de diluir os custos variáveis (SOLANO et al., 2000).

Na porção negativa da mesma dimensão estão as variáveis ligadas à baixa qualidade no ambiente de ordenha (POR:2 e AMB:2), motivação mais abalada do produtor na atividade (VTM:2) e pouca preocupação com a fertilidade das pastagens (ANS:5). Este estado reflete provavelmente uma baixa capacidade de investimento por parte dos produtores, mas também uma carência de gestão do sistema. Damasceno et al. (2008) elencam causas prováveis para este comportamento. Algumas que merecem destaque são; a carência de ações específicas de ATER de acordo com a natureza de cada sistema e a escassez de perspectivas de permanência, emprego e renda na propriedade rural para a garantia da permanência dos mais jovens. Na Figura 2 observa-se a projeção dos casos nos plano fatorial para a tipificação desses segundo as interpretações prévias no tocante às dimensões 1 e 2. Quanto à intensificação da produção leiteira observa-se que, cerca de $11,00 \%$ das propriedades amostradas ( 8 e 6 ) se aproximam de uma condição mais favorável nesse sentido, em torno da intersecção de 0, -3 (Dimensão1, Dimensão2).

Figura 2. Contribuição dos casos, propriedades, $(\mathrm{n}=18)$ para a construção do plano fatorial

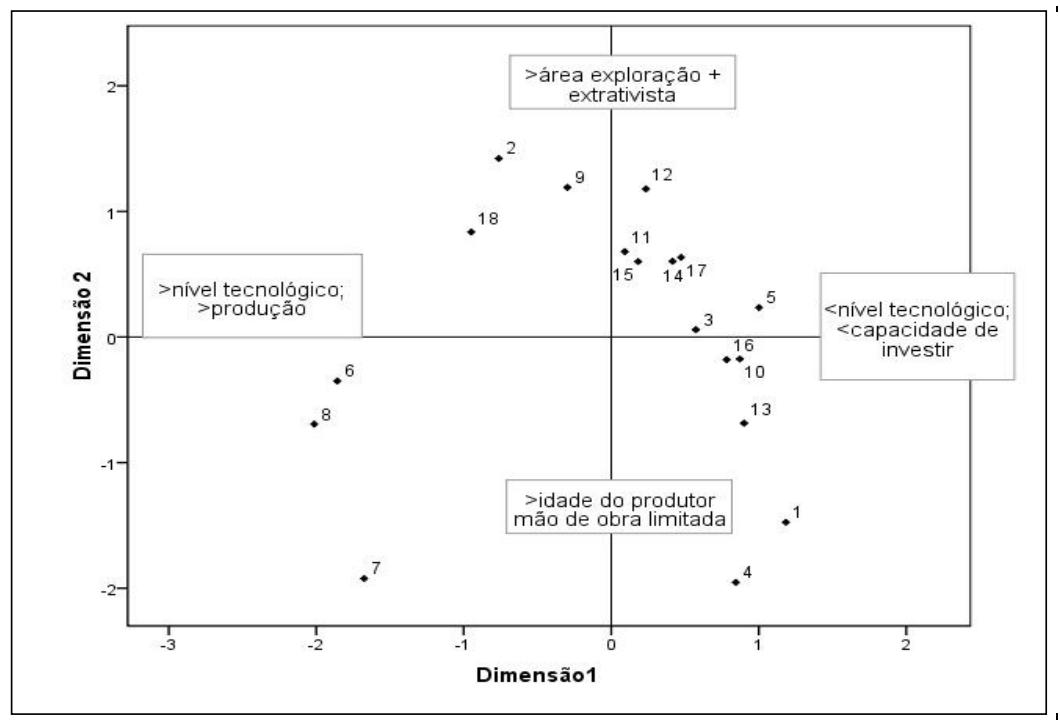

Fonte: Elaboração dos autores.

A maioria está aquém nesse sentido e a trajetória que esses grupos necessitam fazer, ou seja, os pontos de estrangulamento a serem resolvidos em cada sistema são diversos. Para os produtores dos quadrantes na porção positiva da dimensão 2 o problema a ser equacionado é a intensificação de uso dos fatores de produção, principalmente a área. Assim como no trabalho realizado por Hostiou et al. (2006) ficou explícita uma relação entre especialização na produção leiteira e menores áreas de produção. Essa lógica se manteve embora as escalas do que seria uma propriedade grande no estudo daquela autora ( 328,0 ha) e no presente estudo (36,0 ha) discrepem grandemente entre si.

Os sistemas de produção 1 e 4 se encontram em uma situação preocupante em termos de produção, capacidade de trabalho e perenização da atividade. Para esses sistemas, bem como para os sistemas 
10, 16 e 13 (que migram para a mesma condição) as ações de ATER tem de ser mais incisivas e imediatas. Um dos fatores de difícil alcance em termos de intervenção é o da idade do produtor. Esse problema deve ser atacado em médio e longo prazo por meio de políticas públicas de renda e infra-estrutura que possibilitem a perenização desses sistemas via fixação de pessoas mais jovens, sob pena do desaparecimento desses sistemas, como apontado por Damasceno et al. (2008).

Para explicitar os grupos as variáveis foram submetidas a uma classificação Hierárquica
Ascendente ou "Clusters analisys" (LEBART; MORINEAU; TABARD, 2000) apresentada na Figura 3.

A finalidade da análise de Classificação Hierárquica é agrupar semelhantes de forma a manter máxima variância entre os grupos e baixa variância intra-grupos. Neste caso foram utilizadas as mesmas variáveis da ACM e os resultados foram a formação de 4 grupos por similaridades de comportamento.

Figura 3. Gráfico de estalactites proveniente da formação dos grupos de produtores (cluster), baseado na distância Euclidiana entre os casos, dada pelas variáveis originais

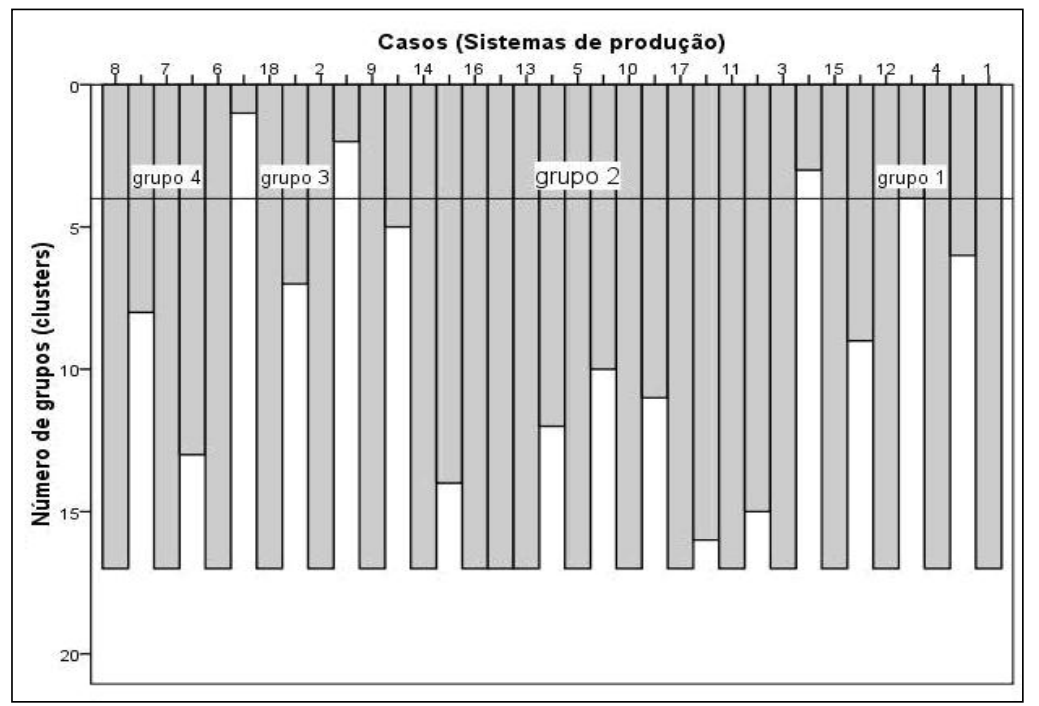

Fonte: Elaboração dos autores.

Os sistemas que fizeram parte de cada grupo (cluster) foram: 6, 7 e 8 (Grupo 4), 2 e 18 (Grupo 3), 3, 5, 9, 10, 11, 13, 14, 16, 17 (Grupo 2) e 1, 4, 12, 15 (Grupo1). Essa divisão permite a testar as variáveis de caracterização da ACM contra as variáveis de qualidade do leite (resposta) uma vez que se utilizam os grupos como "tratamentos", onde as ações se dão de maneira mais homogênea. Desta forma procedeu-se uma análise de variância como apresentado na Tabela 5.
Os grupos 1 e 2 não apresentaram diferenças entre si para as variáveis de produção e qualidade do leite. Os as demais comparações entre grupos exceto entre o grupo 3 e 4 apresentaram efeito para a média de produção média diária por animal $(\mathrm{P}<0,05)$. A magnitude desse efeito foi de 8,17 ;

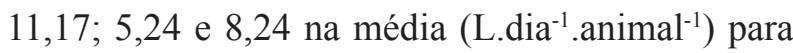
as comparações entre os grupos: 1 e $2 ; 1$ e $3 ; 1$ e 4; 2 e 3 e entre os grupos 2 e 4, respectivamente. As maiores diferenças promovidas pelas 
comparações dos demais grupos com os grupos 3 e 4 são provavelmente resultantes de sistemas mais intensificados de alimentação como observado por Smith, Moreira e Latrille (2002) estudando sistemas de produção leiteiros na $5^{\mathrm{a}}$ região do Chile.

Os grupos 2 e 4, comparados entre si tiveram uma diferença da ordem de $6,08 \mathrm{mg} \cdot \mathrm{dL}^{-1}$ para o nitrogênio uréico no leite - NUL, a única que apresentou importância estatística. Provavelmente esta foi provocada em conseqüência do grupo 4 reunir os sistemas com maior "input" tecnológico, bem como consumo de proteína proveniente de concentrados na dieta ou fontes não protéicas como a ureia e cana. Mesmo assim o valor médio para NUL para os rebanhos pertencentes ao grupo 4 ficou em torno de 14,0 mg.dL $\mathrm{dL}^{-1}$, abaixo do nível considerado problemático para a reprodução (RAJALA-SCHULTZ et al., 2001).

Tabela 5. Comparação das diferenças entre os grupos de produtores (clusters) para as variáveis respostas de qualidade e produção do leite por meio de Análise de Variância - Anova

\begin{tabular}{lccrrrrc}
\hline Grupos & $\mathbf{1 ~ e ~ 2}$ & $\mathbf{1 ~ e ~ 3}$ & $\mathbf{1 ~ e ~ 4}$ & $\mathbf{2 ~ e ~ 3}$ & $\mathbf{2 ~ e ~ 4}$ & $\mathbf{3 ~ e ~ 4}$ & ANOVA $^{\mathbf{a}}$ \\
\hline PROD & 2,927 & $8,166^{*}$ & $11,171^{*}$ & $5,238^{*}$ & $8,243^{*}$ & 3,005 & 0,001 \\
GORD & $-0,264$ & $-0,133$ & $-0,174$ & 0,131 & 0,09 & $-0,041$ & 0,788 \\
PROT & $-0,056$ & $-0,205$ & 0,019 & $-0,150$ & 0,075 & 0,224 & 0,447 \\
LACT & 0,283 & $-0,181$ & 0,410 & $-0,464$ & 0,127 & 0,591 & 0,770 \\
ST & $-0,376$ & $-0,816$ & $-0,253$ & $-0,440$ & 0,123 & 0,563 & 0,180 \\
CCS & 278,484 & 423,731 & 102,783 & 145,247 & $-175,701$ & $-320,948$ & 0,455 \\
NUL & $-0,31$ & $-1,129$ & 5,770 & $-0,819$ & $6,080^{*}$ & 6,899 & 0,054 \\
\hline
\end{tabular}

PROD = representa a produção média por animal $\left(\right.$ L.animal ${ }^{-1} \cdot$ dia $\left.^{-1}\right)$ de cada propriedade; GOR = Gordura $(\%)$; PROT = Proteína $(\%) ;$ LACT $=$ lactose (\%); ST = Sólidos totais (\%); CCS = Contagem de Células Somáticas (1x 10 $)$; NUL = Nitrogênio uréico no leite $\left(\mathrm{mg} . \mathrm{dL}^{-1}\right)$; a: Valores de significância para análise de variância que testou a diferença entre os clusters; *significativo $(\mathrm{P}<0,05)$ para o teste Tukey.

Fonte: Elaboração dos autores.

\section{Conclusões}

Foram identificados grupos distintos quanto à gestão zootécnica permitindo inferir de forma robusta sobre as características dos sistemas de produção afetando os resultados da atividade leiteira, a qualidade e produção do leite.

A produção foi à variável que mais respondeu à diversidade dos grupos, sugerindo que as ações de ATER nos sistemas leiteiros devem ser direcionadas de acordo com os gargalos de cada sistema.

As variáveis ligadas à qualidade sanitária não foram afetadas pelos grupos provavelmente devido à restrição amostral. A ampliação do estudo em número de sistemas provavelmente poderá responder de maneira mais incisiva a esse fato.

\section{Referências}

BARROSO, L. P.; ARTES, R. Análise multivariada. Lavras: UFLA, 2003. $151 \mathrm{p}$.

BRASIL. Ministério da Agricultura, Pecuária e Abastecimento - MAPA. Regulamento Técnico de identidade e qualidade de leite cru refrigerado. In: BRASIL. Ministério da Agricultura, pecuária e Abastecimento. Instrução normative $\mathrm{n}^{\circ} 51$, de 18 de setembro de 2002. Diário Oficial [da] União, 20 set. 2002. Seção 1, p. 13.

DAMASCENO, J. C.; BOUNDERMULLER FILHO, A.; RAMOS, C. E. C. O.; SANTOS, J. C.; SANTOS, G. T. dos. Papel do homem na gestão e controle de qualidade da produção de leite. In: SANTOS, G. T.; UHLIG, L., BRANCO, A. F.; JOBIM, C. C.; DAMASCENO, J. C.; CECATO, U. (Ed.). Inovação tecnológica na cadeia produtiva do leite e a sustentabilidade da pecuária leiteira. Maringá: Eduem, p. 271-284, 2008. 
HOSTIOU, N.; HOSTIOU, N.; VEIGA, J. B.; TOURRAND, J. F. Dinâmica e evolução de sistemas familiares de produção leiteira em Uruará, frente de colonização da Amazônia brasileira. Revista de Economia e Sociologia Rural, Brasília, v. 44, n. 2, p. 295-311, 2006.

LEBART, L.; MORINEAU, A.; TABARD, N. Statistique exploratoire multidimensionnelle. $3^{\mathrm{e} m}$ ed. Paris: Dunod, 2000.

RAJALA-SCHULTZ, P. J.; SAVILLE, W. J. A.; FRAZER, G. S.; WITTUM, T. E. Association between milk urea nitrogen and fertility in Ohio dairy cows. Journal of Dairy Science, Savoy, v. 84, n. 2, p. 482-489, 2001.

SMITH, R. R.; MOREIRA, V.; LATRILLE, L. Caracterización de sistemas productivos lecheros en la $\mathrm{X}$ región de Chile mediante análisis multivariable. Agricultura Técnica, Santiago, v. 62, 375-395, 2002.

SOLANO, C.; BERNUÉS, A.; ROJAS, F.; JOAQUÍN, N.; FERNANDEZ, W.; HERRERO, M. Relationships between management intensity and structural and social variables in dairy and dual-purpose systems in Santa Cruz, Bolivia. Agricultural Systems, Amsterdam, v. 65, n. 3, p. 159-177, 2000.

SOLANO, C.; LEÓN, H.; PÉREZ, E.; HERRERO, M. Who makes farming decisions? A study of Costa Rican dairy farmers. Agricultural Systems, Amsterdam, v. 67, n. 3, p. 181-199, 2001.
SOUZA, G. L.; SANTOS, G. T.; SAKAGUTI, E. S.; DAMASCENO, J. C.; MATSUHITA, M.; HORST, J. A.; VILLALBA, R. G. Avaliação da composição do leite UHT proveniente de dois laticínios das regiões Norte e Noroeste do Estado do Paraná. Acta Scientiarum Animal Sciences, Maringá, v. 26, n. 2, p. 259-264, 2004.

TRONCO, V. M. Manual para a inspeção da qualidade do leite. 3. ed. Santa Maria, RS: UFSM, 2008.

VALLADARES, L. Os dez mandamentos da observação participante. Revista Brasileira de Ciência Social, São Paulo, v. 22, n. 63, p.153-155, 2007.

VERDEJO, M. E. Diagnóstico rural participativo; guia prático DRP. Brasília: Secretaria da Agricultura Familiar, MDA. 2006. 62 p.

VILELA, D.; LEITE, J. L. B.; RESENDE, J. C. Políticas para o leite no Brasil: passado, presente e futuro. In: SIMPÓSIO SOBRE SUSTENTABILIDADE DA PECUÁRIA LEITEIRANA REGIÃO SUL DO BRASIL, 1., 2002, Maringá. Anais... Maringá: UEM/CCA/DZO/ NUPEL, 2002. p. 1-26.

ZOCCAL, R.; GOMES, A. T. Zoneamento da produção de leite. In: ZOCCAL, R.; CARVALHO, L. A.; MARTINS, P. C.; ARCURI, P. B.; MOREIRA, M. S. P. (Ed.). A inserção do Brasil no mercado internacional de lácteos. Juiz de Fora: Embrapa Gado de Leite, 2005. p. 163-180. 